Trieste

\title{
QUESTIONI MINORI DI LINGUA E CULTURA EGIZIANA
}

1. Il graffito Sakkara T (riedito in K. A. Kitchen, Ramess. Inscript. 3, 438), datato all'anno 48 del regno di Ramesse II, è piuttosto interessante: iscritto sulle pareti di uno degli edifici del complesso funerario del Faraone Djoser, esso è composto da due testi distinti (A: $1-4 ; \mathrm{B}: 5-7)$, redatti da due persone diverse che forse sono andate assieme ${ }^{1}$ per turismo culturale o religioso a Sakkara. B è costituito da una serie di auguri funebri piuttosto banali, mentre A, molto mal conservato, pone problemi esegetici fastidiosi. Dopo la datazione e la citazione del nome del redattore, compare un poco comprensibile $] h r y w t s-R^{\mathrm{e}}[$, che il primo editore del testo ha ritenuto equivalente alla designazione della necropoli. Alla riga 4 si legge

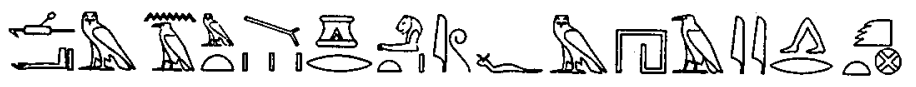

Alla frase segue un'esortazione etica: «Non essere parziale fintanto che sei sulla terra; non ci si può proteggere dal momento[ della morte?......]», la cui seconda parte - se la capisco bene - è morfosintatticamente scorretta. La frase sopra riportata è preceduta da una lunga lacuna ed è, per contro, inequivoca: «[...] uno dei morti del passato che è sceso in Egitto». La frase è imbarazzante proprio perché è trasparente: con 'Egitto' (letter.: (Terra) Nera) si intende la valle del Nilo insediata e coltivata, per cui il 'discendere' è il letterale discendere dalle terre desertiche della riva occidentale, ossia in questo caso dall'altopiano di Sakkara. Ne consegue che lo spirito di un morto del passato si era manifestato al redattore del testo $\mathrm{A}$ e si può presumere che la visita a Sakkara avesse proprio il fine di pacificare il defunto senza pace: ciò spiegherebbe bene anche la natura dell'esortazione finale ed i voti funerari espressi dal testo B.

1 B non è datato e non presenta alcuna introduzione. 
Manifestazioni di spiriti di trapassati sono ben note, come mostra il celebre racconto neo-egiziano di Khonsuemhab e lo spettro.

2. Il sistema geroglifico egiziano di età tarda vide, come è noto, uno straordinario rigoglio di segni i cui valori fonetici erano basati non solo sulle nuove realtà fonetiche della lingua, ma anche su veri e propri giochi di segni, allusioni grafico-simboliche e giochi di parole. Un segno è particolarmente curioso: esso rappresenta un uomo che tiene per la coda un ippopotamo o, piú spesso, un maiale ed equivale alla parola hśb $\underline{\underline{d}}$ «lapislazuli», attestata anche nelle forme hssbd e, con metatesi molto frequente, hsśdb.

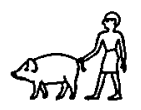

Ma perché si è giunti a tale valore fonetico? Le proposte sinora avanzate (Goodwin «ZÄS» 6 p. 7; Fairman, «ASAE» 43 p. 108) hanno il merito di aver compreso che si tratta di un gioco paretimologico con la parola $d b$ «ippopotamo», ma non sono procedute credibilmente oltre. E ciò perché hanno presunto che il gesto dell'uomo nel gruppo geroglifico fosse quello di 'respingere, allontanare' l'animale, ed è curioso perché è assolutamente chiaro che l'uomo trattiene l'animale per la coda. Non sappiamo quale fosse la pronuncia delle parole egiziane $d b$ e $h s ́ d b$, e tuttavia possiamo farci un'idea del processo paretimologico: $d b$ doveva essersi evoluto in ${ }^{*} t^{\prime} p$, che evidentemente assonava con la finale della parola per «lapislazuli», per cui dobbiamo identificare una verbo egiziano con struttura consonantica $*_{s ̌ s}\left(h\right.$ si evolve quasi regolarmente in $\tilde{s}$ ) o $*_{s ̌ s}$ (la dentale finale sarebbe stata comunque fusa al fonema iniziale della parola ${ }^{*} t^{t} p$ ) e con il significato di «trattenere». L'unico candidato che soddisfi le condizioni proposte mi

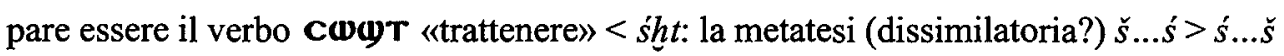
è altrettanto comune. Il geroglifico va dunque 'letto' «trattenere l'ippopotamo».

3. Il lemma 'A $\theta \rho \imath \beta i \zeta$ dell'Etymologicum Magnum 387 è testualmente corrotto. Questo è il testo:

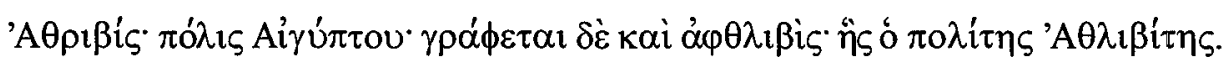

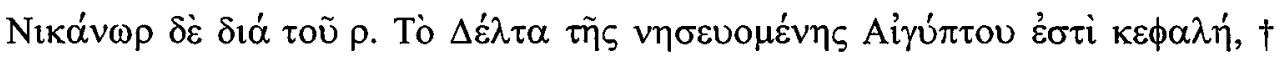




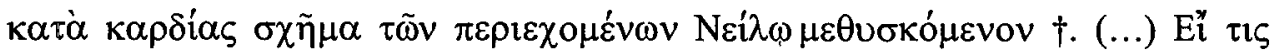

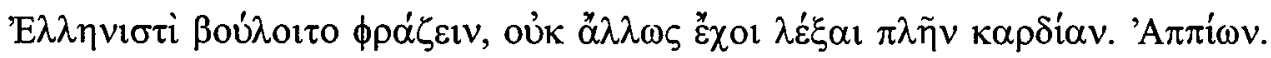

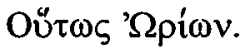

Il corrispondente passo dell'Etymologicum Symeonis (206) è altrettanto corrotto, ma il senso generale del lemma si coglie facilmente.

Athribis (egiz. $h w t-h r y-j b$ ed $h w t-t^{3}-h r y-j b$; v. oltre) è nome di città dell'Egitto: nel copto il nome è attestato in forme molto simili, дөрHвє ed дөגHвє che giustificano le due rese greche. Il lemma dell'Etym. è basato sull'autorità di Apione (FGH 616 F 9) via Orione, il quale evidentemente aveva istituito, certamente su ispirazione di genuine fonti egiziane, una comparazione tra la posizione geografica di Athribi e quella del cuore: il Delta alluvionale dell'Egitto sarebbe stata la testa, Athribi invece, secondo lo schema corporale, sarebbe stato il cuore della regione bagnata dal Nilo. Mi pare altrettanto sicuro che nel testo doveva trovarsi un'esegesi etimologica, poiché solo in questo caso si spiega l'ultima frase: «Se si volesse dirlo in greco, non si potrebbe esprimere diversamente che con 'cuore'».

Il nome Athribi è attestato nei testi egiziani antichi nelle forme $\mathrm{G}[\mathrm{\square}$ mora del centro» ed ben si addicono al testo del lemma: inoltre in egiziano l'espressione 'che sta in mezzo' vale letteralmente 'che sta sul cuore ( $h r y-j b)$ '. Come si vede, Apione aveva ragione: d'altronde è noto che egli era di lingua madre e di cultura egiziana pur se di paideia greca.

4. Giulio Sesto Africano, autore del II-III sec. della nostra era, ci conserva una parola egiziana che credo sia sfuggita agli studiosi. Nei suoi Cesti $(4,1)$ egli parla delle

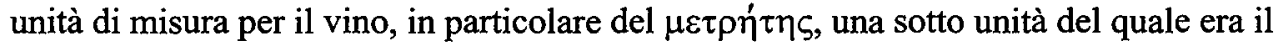
$\xi \dot{\varepsilon} \sigma \tau \eta \varsigma:$ appunto di quest'ultima egli dice che gli Egiziani la chiamano ívıov, il che corrisponde al vero. La voce è un adattamento grammaticalizzato di *hin, conservato dal copto (sah.) 2IN «vaso; misura per liquidi», demotico $h n$, egiz. ant. $h n w$, passato anticamente anche all'ebraico (היו) ed all'accadico (hina); la voce è attestata anche nei papiri documentari greci d'Egitto. 


\section{Povzetek}

NEKAJ DROBNIH VPRAŠANJ EGIPČANSKEGA JEZIKA IN KULTURE

1. Egipčanski grafit poroča o prikazni duhá; 2. ljudska etimologija nekega egipčanskega hieroglifa; 3. egipčanski etimon imena mesta Athribis je bil znan že grški leksikografiji; 4. egipčanska izposojenka v jeziku rimskega zgodovinarja iz 3. st. Julija Siksta Afričana. 